\title{
Perilipin-related protein regulates lipid metabolism in $C$. elegans
}

Ahmed A. Chughtai, Filip Kaššák, Markéta Kostrouchová, Jan Philipp Novotný, Michael W. Krause, Vladimír Saudek, Zdenek Kostrouch, Marta Kostrouchová

Perilipins are lipid droplet surface proteins that contribute to fat metabolism by controlling the access of lipids to lipolytic enzymes. Perilipins have been identified in organisms as diverse as metazoa, fungi, and amoebas but strikingly not in nematodes. Here we identify the protein encoded by the W01A8.1 gene in Caenorhabditis elegans as the closest homologue and likely orthologue of metazoan perilipin. We demonstrate that nematode W01A8.1 is a cytoplasmic protein residing on lipid droplets similarly as human perilipins 1 and 2. Downregulation or elimination of W01A8.1 affects the appearance of lipid droplets resulting in the formation of large lipid droplets localized around the dividing nucleus during the early zygotic divisions. Visualization of lipid containing structures by CARS microscopy in vivo showed that lipid-containing structures become gradually enlarged during oogenesis and relocate during the first zygotic division around the dividing nucleus. In mutant embryos, the lipid containing structures show defective intracellular distribution in subsequent embryonic divisions and become gradually smaller during further development. In contrast to embryos, lipid-containing structures in enterocytes and in epidermal cells of adult animals are smaller in mutants than in wild type animals. Our results demonstrate the existence of a perilipin-related regulation of fat metabolism in nematodes and provide new possibilities for functional studies of lipid metabolism. 


\section{Perilipin-related protein regulates lipid metabolism in $C$. elegans}

2 Ahmed Ali Chughtai ${ }^{1}$, Filip Kaššák ${ }^{1}$, Markéta Kostrouchová1,2, Jan Philipp Novotný1,

3 Michael W. Krause ${ }^{3}$, Vladimír Saudek ${ }^{4, \#}, Z_{\text {Zdenek Kostrouch }}{ }^{1}$ and Marta Kostrouchová ${ }^{1, *}$

61 Institute of Cellular Biology and Pathology, First Faculty of Medicine, Charles University in

7 Prague, Albertov 4, 12800 Prague 2, Czech Republic

82 Department of Pathology, Third Faculty of Medicine, Charles University in Prague, Ruská 87,

910000 Prague 10, Czech Republic

103 Laboratory of Molecular Biology, National Institute of Diabetes and Digestive and Kidney

11 Diseases, National Institutes of Health, Bethesda, Maryland, USA

124 University of Cambridge Metabolic Research Laboratories, Wellcome Trust-Medical Research

13 Council Institute of Metabolic Science, Cambridge CB2 0QQ, United Kingdom

$15{ }^{*}$ Corresponding author

16 " Corresponding author for bioinformatics

18 * Address for correspondence: Marta Kostrouchová, Institute of Cellular Biology and Pathology,

19 First Faculty of Medicine, Charles University in Prague, Ke Karlovu 2, 12800 Prague 2, Czech

20 Republic. Email: marta.kostrouchova@1f1.cuni.cz

21 \# Address for correspondence concerning bioinformatics: Vladimir Saudek, email:

22 vs317@cam.ac.uk

24 Running title: Perilipin homologue in C. elegans 


\section{Abstract}

26 Perilipins are lipid droplet surface proteins that contribute to fat metabolism by controlling the 27 access of lipids to lipolytic enzymes. Perilipins have been identified in organisms as diverse as 28 metazoa, fungi, and amoebas but strikingly not in nematodes. Here we identify the protein encoded by the W01A8.1 gene in Caenorhabditis elegans as the closest homologue and likely orthologue of metazoan perilipin. We demonstrate that nematode W01A8.1 is a cytoplasmic

31 protein residing on lipid droplets similarly as human perilipins 1 and 2. Downregulation or

32 elimination of W01A8.1 affects the appearance of lipid droplets resulting in the formation of 33 large lipid droplets localized around the dividing nucleus during the early zygotic divisions. 34 Visualization of lipid containing structures by CARS microscopy in vivo showed that lipidcontaining structures become gradually enlarged during oogenesis and relocate during the first

36 zygotic division around the dividing nucleus. In mutant embryos, the lipid containing structures 37 show defective intracellular distribution in subsequent embryonic divisions and become 38 gradually smaller during further development. In contrast to embryos, lipid-containing structures in enterocytes and in epidermal cells of adult animals are smaller in mutants than in wild type animals. Our results demonstrate the existence of a perilipin-related regulation of fat metabolism in nematodes and provide new possibilities for functional studies of lipid metabolism.

Keywords Caenorhabditis elegans, development, fat metabolism, lipid droplets, perilipin Abbreviations LD - lipid droplet, W01A8.1 - cosmid gene name, W01A8.1(a, b, or $c)-$ transcript isoforms encoded by W01A8.1, W01A8.1(a, b, or c) - proteins encoded by corresponding transcripts 


\section{Introduction}

Perilipins are regulatory proteins targeted to the surface of fat storage organelles lipid dropplets (LDs) where they contribute to the regulation of lipid metabolism (Brasaemle 2007). Functional perilipins (PLIN proteins encoded by the PLIN genes) (Lu et al. 2001) have been identified in very diverse organisms such as Drosophila (Teixeira et al. 2003), Dictyostelium (Du et al. 2013) and fungi (Wang \& St Leger 2007) and protein databases list clear orthologues in diverse, non-plant eukaryota, including the simplest metazoan Trichoplax adherens, sponges, crustaceans, and choanoflagelates (UniProt proteins B3RRM2, I1GA14, G5DCP6, F2UJD9, respectively). In humans and other mammals, the PLIN family consists of five members (Kimmel et al. 2010) (Perilipin 1 to 5) with diverse tissue distribution, specificity, and partially redundant functions. Strikingly, no perilipin othologue has been identified in C. elegans, suggesting that nematode-specific lipid regulatory pathways might exist in this phylum and perhaps in others as well.

This unusual evolutionary gap in the perilipins prompted us to re-examine the C. elegans genome for a gene related to mammalian perilipin. We identify $W 01 A 8.1$ as the likely C. elegans orthologue of mammalian perilipin genes. We show that W01A8.1 is the previously unrecognized $C$. elegans homologue of vertebrate perilipins that possesses all functional domains characteristic for perilipins and functions in lipid metabolism at the level of lipid droplets.

The protein encoded by W01A8.1 in C. elegans is identified as Mediator Complex subunit 28 (MDT-28) in many protein databases (e.g. Pfam, UniProt, PIR, WormPep) (accessed on March 14, 2015), but the bioinformatics analysis reveals that this is a misannotation. We observe that protein isoforms expressed from W01A8.1 are cytoplasmic proteins, residing predominantly on membranous structures of enterocytes and epidermal cells that have the 
71 characteristics of lipid droplets. We also show that transgene-encoded GFP fusion proteins of

72 human Perilipins 1 and 2 localize in C. elegans similarly as W01A8.1::GFP on vesicular

73 structures that are positive for lipid content. Furthermore, down regulation of W01A8.1 by RNAi

74 or its elimination lead to altered appearance and behavior of lipid droplets prominently observed

75 in the germline and in early embryos. Our results indicate that $C$. elegans can compensate for the

76 loss of W01A8.1 in all developmental stages except early embryos likely by additional fat 77 degradation mechanisms.

78 Our data demonstrates that the perilipin-related regulation of fat metabolism is conserved

79 in C. elegans, and provides a novel insight into early embryonic lipid management. This

80 discovery offers promising possibilities for functional studies of lipid metabolism in a nematode

81 model system.

82

83 Materials and methods

84 Sequence analysis

85 Perilipin orthologues and W01A8.1 sequences were extracted from UniProt, NCBI and

86 OMA (omabrowser.org) databases. Chordate and nematode sequences were aligned separately

87 using the T-Coffee algorithm (Notredame, Higgins \& Heringa 2000) (server tcoffee.crg.cat) and

88 submitted to PSI-BLAST (Altschul et al. 1997) (E-value inclusion threshold $<10^{-3}, 5$ iterations)

89 and HHpred (Remmert et al. 2011; Biegert \& Soding 2008) searches as implemented in

90 MPItoolkit (toolkit.tuebingen.mpg.de). Repeat detection used HHrepID module in MPItoolkit.

91 Alignments were displayed and analyzed in Jalview app (jalview.org).

93 Strains, transgenic lines and genome editing 
Wild type animals, N2 (var. Bristol), were used unless otherwise noted and all strains

95 were maintained as described (Brenner 1974). Transgenic lines were prepared using 96 microinjections into gonads of young adult N2 hermaphrodites as described (Tabara et al. 1999;

97 Timmons, Court \& Fire 2001; Vohanka et al. 2010). All injections also included mCherry co98 injection markers: pCFJ90, pCJ104 and pGH8 (Dickinson et al. 2013).

99 To create mutants, we employed CRISPR/Cas9 system as described (Dickinson et al. 100 2013). The following plasmids were constructed: pCK001 targeting the sgRNA (+323) to the 101 second exon of the W01A8.1 gene (forward primer \#7992), and pCK023 targeting the sgRNA 102 (+1372) to the sixth exon (forward primer \#8078). The reverse primer was \#7993. A scheme of 103 known expressed isoforms listed in WormBase WS246 and the strategy for the disruption of 104 W01A8.1 gene is shown in Fig. S1 and Fig. S2. Primers used in this study are listed in Table S1.

105 The following transgenic lines regulated by W01A8.1 natural promoter were prepared:

106 W01A8.1a/c::gfp and W01A8.1b::gfp (containing the whole coding sequence of isoforms a and 107 b). W01A8.1 isoforms a and c have identical 3' ends which both could be expressed from 108 W01A8.1a/c::gfp. This construct also includes complete untagged isoform $\mathrm{b}$. The GFP-tagged 109 isoform a (plasmid pCK28 $\left\{P_{W 01 A 8.1}:\right.$ W01A8.1(a)synth::gfp::unc-54 3'UTR $\}$ ) was constructed 110 by synthesizing the W01A8.1a sequence with modified codons to allow protection from 111 CRISPR/Cas9 targeted sgRNA and prepared as a GeneArt ${ }^{\circledR}$ Strings $^{\text {TM }}$ DNA Fragment from 112 Invitrogen (Invitrogen, Carlsbad, Ca, USA) and cloned using GeneArt ${ }^{\circledR}$ Seamless Cloning 113 System (Invitrogen) into pPD95.75(NeoR). Schemes for isoforms expressed from W01A8.1 gene 114 and preparation of GFP tagged transgenes are given in Figs. S1 and S2.

115 Human PLIN2 and PLIN3 were cloned from a collection of anonymous unmarked 116 samples (PLIN2), and from human peripheral lymphocytes (PLIN3) donated by a volunteer with 
117 a written consent in compliance with the legislation of the Czech Republic and European Union

118 (Act No 372/2011 of 11. 11. 2011 on Health Care Services, Coll., Paragraph 81, section 1a and

119 section 4a, which is in accordance with the declaration of Helsinki) and was approved by the

120 Ethics Committee of the First Faculty of Medicine, Charles University in Prague (Ref. No.

121 MZ13-UK1LF-KostrouchZdenek). Human PLIN1 optimized for C. elegans was prepared as a

122 synthetic sequence requested as a GeneArt ${ }^{\circledR}$ Strings $^{\mathrm{TM}}$ DNA Fragment from Invitrogen ${ }^{\mathrm{TM}}$.

123 Transgenic lines expressing human PLIN1, PLIN2, PLIN3 tagged by GFP under W01A8.1

124 natural promoter were prepared using N2 animals and animals with disrupted W01A8.1. Primers

125 used for cloning PLIN2 and PLIN3 are listed in supplementary table S1.

126

127 Downregulation of gene expression by RNA interference

128 Downregulation of W01A8.1 expression used the RNAi protocol of injection of dsRNA

129 into gonads of young adult hermaphrodites as well as RNAi through feeding animals bacteria

130 producing dsRNA as previously described (Tabara et al. 1999; Timmons, Court \& Fire 2001;

131 Vohanka et al. 2010).

132

133 Injection RNAi Protocol

134 Double stranded RNA (dsRNA) was prepared for injection by in vitro transcription

135 reactions (SP6/T7 Riboprobe ${ }^{\circledR}$ in vitro Transcription Systems, Promega, Madison, WI, USA)

136 from opposing promoters and subsequent annealing of each single stranded RNA (ssRNA)

137 product prior to injection. For RNAi directed against W01A8.1, BamHI or ApaI linearized

138 pCK014 plasmid preparations were used in separate reactions to generate complementary

139 ssRNA. After linearization the DNA was phenol-chloroform extracted and ethanol precipitated. 
140 BamHI linearized DNA was transcribed using T7 RNA Polymerase while ApaI linearized DNA

141 with SP6 RNA Polymerase. After in vitro transcription ( $\sim 2$ hours) equal volumes of sense and

142 antisense RNA were incubated at $75^{\circ} \mathrm{C}$ for $10 \mathrm{~min}$ and then cooled at room temperature for 30

143 min. Control RNAi was prepared from the promoter region of $n h r-60$ as previously described

144 (Simeckova et al. 2007). The dsRNA concentration was measured using a UV spectrophotometer

145 and $\sim 1 \mu \mathrm{g} / \mu \mathrm{l}$ was used for injections.

147 Feeding RNAi Protocol

148 Nematode Growth Medium (NGM) agar plates were prepared according to standard

149 protocols and were supplemented with Ampicillin (100 $\mu \mathrm{g} / \mathrm{ml}$ final concentration) and isopropyl

$150 \beta$-D-1-thiogalactopyranoside (IPTG) (1.5 mM final concentration). E. coli strain HT115 was

151 transformed with pCK015 and control L4440 vector. After transformation, a single colony from

152 each was used to inoculate LB medium with Ampicillin (100 $\mu \mathrm{g} / \mathrm{ml}$ final concentration). The

153 culture was grown to $\mathrm{OD}_{600} \approx 1.0 ; 900 \mu \mathrm{l}$ of culture was poured onto NGM agar plates to

154 completely cover the surface and $750 \mu \mathrm{l}$ of the suspension was removed to leave $150 \mu 1$ of the

155 suspension on the plates. The bacteria were allowed to grow and were induced overnight at room

156 temperate $\left(\sim 22^{\circ} \mathrm{C}\right)$.

157

158 Fecundity and brood size assay

159 Fecundity measurement following RNAi (injection method) was conducted using a total 160 of 50 young adult worms ( 25 control and 25 inhibited by RNAi specific for W01A8.1). Progeny

161 was counted 24 hours and again 48 hours after injections. Brood size assay was performed for 
162 W01A8.1 disrupted animals and controls ( $\mathrm{n}=15$ for each group). The progeny was determined

163 during 6 days. The experiments were conducted at room temperature $\sim 22^{\circ} \mathrm{C}$.

164 Fecundity measurement after RNAi using feeding protocol was performed over two

165 generations to maximize the effect of knockdown. For this, a semi-synchronized population was

166 isolated using standard WormBook (http://www.wormbook.org/) bleaching protocol. Hatched L1

167 stage worms were placed on NGM agar RNAi (W01A8.1 specific and control) plates at $\sim 22^{\circ} \mathrm{C}$.

168 Small, synchronized populations, of parents (P0) were transferred to fresh RNAi plates and

169 allowed to lay progeny (F1). F1 generation animals were transferred to new RNAi plates and F2

170 generation was scored for a total of $21 \mathrm{~F} 1$ parents in each group. The experiment was repeated

171 twice to confirm the results.

173 RNA isolation and cDNA synthesis Total RNA was extracted as previously described (Vohanka et al. 2010). Briefly, $C$.

175 elegans washed, pelleted and re-suspended in re-suspension buffer with proteinase K. After lysis

176 TRIzol® Reagent (Invitrogen) was added to the mixture and the standard manufacturer's

177 protocol was followed to obtain total RNA. Samples were then treated with DNaseI (New

178 England Biolabs, Ipswich, MA, USA) and again TRIzol-chloroform extracted (Invitrogen) to 179 obtain DNA free total RNA.

180 Human total RNA was also extracted using TRIzol ${ }^{\circledR}$ Reagent (Invitrogen) from 181 peripheral blood lymphocytes and fat tissue. cDNA was prepared from total RNA by reverse 182 transcription using standard protocols for the SuperScript ${ }^{\circledR}$ III First-Strand Synthesis System 183 (Invitrogen) and oligo(dT) priming. 
185 Transcript quantification total RNA isolated as described above, using the Roche Universal Probe Library technique

188 (Hoffmann-La Roche, Basel, Switzerland). Primers and probes for determination of number of transcripts of W01A8.1 are given in supplementary table S1. Levels of W01A8.1 expression were normalized against ama-1.

\section{Single worm PCR} $\mathrm{mM} \mathrm{KCl}, 2.5 \mathrm{mM} \mathrm{MgCl} 2,0.45 \% \mathrm{NP}-40,0.45 \%$ Tween $20,0.01 \%$ Gelatin and $500 \mu \mathrm{g} / \mathrm{ml}$ fresh proteinase $\mathrm{K}$ ) in a PCR tube. Animals were frozen at $-80{ }^{\circ} \mathrm{C}$ for 5 minutes before placing the tube into a thermal cycler and run under the following conditions: heat to $60{ }^{\circ} \mathrm{C}$ for $60 \mathrm{~min}$ reaction mix $(45 \mu 1)$ targeting the template of choice was added and cycled for $\sim 35$ times with Q5 ${ }^{\circledR}$ Hot Start DNA polymerase (New England Biolabs).

\section{LipidTox staining} modifications. Approximately 200 to 500 animals were harvested from NGM plates with $1 \mathrm{X}$

204 PBS and washed several times to remove E. coli and pelleted at $1,500 \mathrm{x}$ g. To the pellet, $500 \mu 1$ 
206 Spermine, $30 \mathrm{mM}$ NaPIPES $\mathrm{pH}$ 7.4, 0.2\% beta-ME) and $100 \mu \mathrm{l} 20 \%$ paraformaldehyde were 207 added and the volume was adjusted up to $1 \mathrm{ml}$ with $1 \mathrm{X}$ PBS. Inverting the tube several times 208 mixed the worms in solution after which it was allowed to fix for $\sim 60 \mathrm{~min}$ at room temperature 209 with gentle shaking.

After fixation, animals were pelleted at 1,500 $\mathrm{x}$ and washed 3 times with $1 \mathrm{ml}$ Tris- $\mathrm{HCl}$

211 buffer (100 mM, pH 7.4). After the third wash, the supernatant was discarded down to $100 \mu \mathrm{l}$ and $212650 \mu \mathrm{l}$ of Tris-HCL buffer was added followed by $250 \mu \mathrm{l}$ of fresh/frozen reduction buffer (100 $213 \mathrm{mM}$ Tris-Cl $\mathrm{pH} 7.4,40 \mathrm{mM}$ DTT). Worms were then left shaking for $\sim 30 \mathrm{~min}$ at room 214 temperature. After reduction, worms were washed 3 times in 1X PBS. After the final PBS wash, 215 the volume was brought up to $500 \mu \mathrm{l}$ and then $500 \mu \mathrm{l}$ of LipidTox (Red) (1:500 dilution) 216 (Invitrogen) was added to make a final volume of $1 \mathrm{ml}$. The final concentration of 1:1000 217 dilution of LipidTox was used. The worms were left in the dark for at least 60 min with shaking 218 before viewing.

\section{Microinjections}

Microinjections of plasmids, DNA amplicons or dsRNA into gonads of young adult 222 hermaphrodites were done using an Olympus IX70 microscope equipped with a Narishige 223 microinjection system (Olympus, Tokyo, Japan).

Microscopy

Fluorescence microscopy and Nomarski optics microscopy were done using an Olympus 227 BX60 microscope equipped with DP30BW CD camera (Olympus, Tokyo, Japan). 
229 Coherent Anti-Stokes Raman Scattering microscopy (CARS)

230 For label-free CARS imaging the worms were anesthetized with levamisole and placed

231 on a cover glass with a thin layer of $2 \%$ agarose. The CARS images were acquired with a Leica

232 TCS SP8 CARS system (Leica Microsystems, Mannheim, Germany) consisting of a TCS SP8

233 confocal microscope combined with a picoEmerald laser (APE, Berlin, Germany) offering a

234 fixed Stokes laser line of $1064.5 \mathrm{~nm}$ and a tuneable Pump line from an optical parametric

235 oscillator (780 nm - $940 \mathrm{~nm}$ ). A HC PL IRAPO 40x water immersion objective was used for the

236 imaging and CARS signal was detected with a non-descanned photon multiplier tube detector at

237 the transmitted light side. For imaging of $\mathrm{CH}_{2}$ vibration with Raman shift of $2868 \mathrm{~cm}^{-1}$ pump

238 wavelength of $815.5 \mathrm{~nm}$ was used. The CARS signal was selected with a CARS2000 filter cube

239 placed in front of the detector. Adult animals and embryos were completely scanned and

240 recorded as stacks of focal planes. Recordings for quantitative analysis were done at fixed 241 settings for mutant and wild type.

243 Image analysis

244 Single focal planes (containing the highest number of CARS positive structures) from

245 stacks of representative embryos and adult hermaphrodites were selected and analyzed using 246 ImageJ computer program (http://imagej.nih.gov/ij/). Analysis for embryos was done on images

247 of seven different embryos (seven mutant and seven wild type embryos) inside gravid 248 hermaphrodites (only one and two cell early embryonic stages were chosen for comparison).

249 Analysis of adult somatic tissue was performed on distal body region and we compared five 
250 different mutant adults with five different wild type adults. The images selected for analysis have

251 been provided as supplement.

252

253

Automatic particle counting feature of ImageJ program was used for determination of the

254 number and area of CARS positive structures with manual thresholding as described on

255 http://imagej.net/Particle_Analysis. Image area required for analysis was first selected (the area

256 outside the selected zone was cleared) then the image was converted to an 8-bit scale. Manual

257 threshold was applied with settings yielding the biggest number of individually recognizable

258 structures (adult tissue threshold setting range was 36 to 200 and for embryos 11-13 to 200).

259 Overlapping structures were separated using the "Watershed" command and also by manual line

260 draw feature. "Analyze particles" command generated data sets containing the number and area

261 of particles. Microsoft Excel 2003 was used to perform statistical analysis and two-tailed

262 Student's t-test for determining the p-value. Raw data sets are provided as supplement and labels

263 in the Excel tables correspond to the marked images also provided as supplement.

265 Results

266 Identification of a perilipin orthologue in C. elegans

267 We performed BLASTp searches with individual protein sequences of human perilipins

268 that generated no significant hits to Nematoda sequences in the UniProt database, consistent with 269 previous efforts that failed to identify a perilipin-related protein in this phylum. However, when

270 a sequence alignment of chordate perilipins 2 and 3 (OMA database) was submitted as query in

271 PSI-BLAST, the C. elegans protein W01A8.1a (Q23095_CAEEL) was identified as a highly

272 significant hit $\left(E=3 \times 10^{-13}\right)$. A reciprocal PSI-BLAST search with the aligned closest nematode 
273 homologues of W01A8.1a identified chordate perilipins as strong hits with human Perilipin 2

274 (significance score $\mathrm{E}=10^{-53}$ ) appearing in the second iteration of the search. Similarly, HHpred

275 profile-to-profile searches with human perilipin sequences as a query of the C. elegans proteome

276 identified proteins coded by W01A8.1 (a, b or c) and reciprocally W01A8.1a showed profile

277 homology to all human perilipins and the corresponding Pfam (Punta et al. 2012) perilipin

278 profile (PF03036). Each available nematode proteome contained only a single such perilipin-

279 related sequence, in stark contrast to the insect and chordate proteomes that had 2 to 5 perilipin

280 paralogues. A sequence alignment of Plin2 and 3 from two selected vertebrates is compared with

281 their nematode homologues (Fig. 1). Although the sequence-to sequence comparisons are not

282 sufficient to unravel the sequence homology between vertebrate and nematode Plins, the

283 similarity appear clearly in the profile-to sequence (PSI-BLAST) and profile-to-profile (HHpred)

284 searches. We conclude that vertebrate Plins and nematode W01A8.1 are statistically highly 285 significant homologues.

286 The alignment encompasses a substantial part of C. elegans and human sequences (e.g.

$28790 \%$ of W01A8.1 and $87 \%$ of Perilipin 2) and covers all three domains characteristic for 288 perilipins (N-terminal PAT, imperfect amphiphilic 11-mer repeat (Brasaemle 2007) and C289 terminal four-helix bundle (Hickenbottom et al. 2004)) covering approximately amino acids 10290 100, 125-190 and 220-380 respectively in W01A8.1a. As W01A8.1 and human perilipins appear 291 to be the best mutual reciprocal PSI-BLAST and HHpred hits, W01A8.1 is a very good 292 candidate for a C. elegans orthologue of perilipin.

293 Protein databases annotate W01A8.1 as Mediator Complex subunit 28, hence the official 294 protein name assignment of MDT-28 in WormBase (WS246). Pfam database (Punta et al. 2012) 295 based the Mediator 28 Hidden Markov model profile on a seed alignment of bovine and 
296 mosquito Mediator 28 sequences with W01A8.1. This very profile was probably used

297 subsequently in all automatic annotations of the nematode sequences. However, no substantial

298 homology between W01A8.1 and Mediator 28 exists as shown in the above searches. Since

299 using the WormBase name of W01A8.1 (MDT-28) would be misleading, the gene is referred

300 here by the cosmid name W01A8.1, which gives rise to at least three protein isoforms designated

301 W01A8.1a, W01A8.1b, and W01A8.1c from at least seven different transcripts (W01A8.1a.1,

302 W01A8.1a.2, W01A8.1b.1, W01A8.1b.2, W01A8.1b.3, W01A8.1c.1, W01A8.1c.2). The three

303 protein isoforms are 415,385 , and 418 amino acid residues in length for isoform a, b, and c,

304 respectively (Fig. S1). According to the C. elegans nomenclature, we suggest to rename

305 W01A8.1 as Cel-plin-1 (isoform a, b, and c) and proteins Cel-PLIN-1 (isoform a, b, and c).

W01A8.1 protein products are cytoplasmic and reside primarily on lipid droplets

If the proteins encoded by W01A8.1 act as perilipins, they would be expected to be

associated with lipid droplets (Kozusko et al. 2015). To test this, we created translational

reporter transgenes regulated by the putative endogenous promoter expressing isoform $b$ and

311 lines in which the genomic locus was tagged by an in-frame C-terminal GFP cassette. The

312 second transgene, W01A8.1a/c::gfp, is likely to express not only high levels of a and c tagged

313 isoforms, but also the native isoform b (Fig. S1). The translational fusion constructs resulted in

314 high levels of cytoplasmic proteins present in intestinal and epidermal cells on vesicular

315 structures with the characteristic appearance of lipid droplets. This pattern of expression and

316 cellular distribution was observed beginning at the three-fold embryonic stage and continued

317 throughout development to adulthood (Fig. 2). To confirm that the observed GFP-associated

318 vesicular structures were indeed lipid droplets, transgenic animals were stained with the 
319 lipophilic reagent LipidTox as previously described (O'Rourke et al. 2009). The translational

320 GFP fusion protein reporters were localized at the periphery of fat droplets that were LipidTox

321 positive (Fig. 2).

322

323 Human PLINs 1 and 2 label identical compartments as W01A8.1 proteins in $C$.

324 elegans

325 We prepared transgenic C. elegans lines expressing human PLIN1, PLIN2 and PLIN3

326 fused to GFP and regulated by the W01A8.1 promoter. PLIN1::GFP and PLIN2::GFP were

327 localized on spherical cytoplasmic structures primarily in gut and epidermal cells (Fig. 3A, C, D

328 and F) with identical appearance as W01A8.1 translational reporter GFP tagged proteins and

329 Drosophila PLIN1::GFP expressed in C. elegans as reported by Liu et al. (Liu et al. 2014).

330 PLIN3 expression was diffusely cytoplasmic and only faintly defined spherical structures (Fig.

$3313 \mathrm{G}$ and I). The structures clearly labeled with PLIN1::GFP and PLIN2::GFP were also positive

332 in LipidTox staining (shown for PLIN2::GFP in Fig. 3J, K and L). We conclude that W01A8

333 proteins are localized on the same structures as human PLIN1 and PLIN2.

334

335 W01A8.1 reduction-of-function alters the appearance of lipid droplets in early

336 embryos and causes a reduction of brood size

337 To test the function of W01A8.1, we used RNAi done by germline injection and by

338 feeding. W01A8.1 RNAi made by microinjections and feeding resulted in a significantly smaller

339 brood size, with approximately $30 \%$ less progeny. RNAi made by microinjections resulted in

$340 \sim 52 \%$ reduced progeny laid in the first 24 hours after microinjections and after 48 hours $\sim 28 \%$ 
341 reduction in progeny laid compared to controls $(n=260, n=550$, for day one and $n=1000, n=$

3421400 for day two).

343 Repetition of knockdown by RNAi feeding over two generations confirmed this

344 observation (Fig. S4). dsRNA feeding caused the W01A8.1 specific group to produce $\sim 30 \%$ less

345 larvae compared to controls, experiment was repeated twice independently with consistent

346 findings. We confirmed, using RT-qPCR, that feeding based knockdown (represented in Fig. S4)

347 resulted in approximately $45 \%$ decrease in W01A8.1 transcripts (data not shown).

348 Staining of adult hermaphrodites with LipidTox (after formaldehyde fixation) revealed

349 larger lipid droplets in early embryos derived from adults inhibited for W01A8.1 (Fig. 4A and B)

350 compared to controls (Fig. 4C and D).

351

352 Targeted disruption of W01A8.1 results in early embryonic defects but not lethality

353 In order to eliminate the W01A8.1 function completely, we designed a CRISPR/Cas9-

354 mediated gene editing approach to eliminate almost the entire coding region (Fig. S2). We also

355 included a rescuing plasmid consisting of isoform a that was prepared as cDNA synthesized in

356 vitro using synonymous codons (W01A8.1(a)synth::gfp) that is protected against CRISPR/Cas9

357 targeted editing but allows the production of the wild type isoform a at the protein level. Lines

358 that expressed the GFP fusion transgene were morphologically normal and

359 W01A8.1(a)synth::GFP was found on lipid droplet-like structures as expected (Fig. 4E and G)

360 that also stained positive by LipidTox (Fig. 4F and G). This transgenic strain yielded lines either

361 carrying or losing the rescuing transgene in the background of a disrupted endogenous W01A8.1.

362 The elimination of W01A8.1 was easily monitored by PCR (Fig. S3). Surprisingly, animals with

363 the deleted W01A8.1 locus that lost the extra-chromosomal rescuing array were able to reproduce 
364 normally. From several lines that had a confirmed disruption of W01A8.1 and a confirmed loss of

365 the extrachromosomal array, the line CK123 (KV001) was selected and used for subsequent

366 analyses. As was observed in W01A8.1 RNAi embryos, loss of W01A8.1 activity resulted in the

367 formation of large LipidTox-positive structures (Fig. 4H and I) that were clearly bigger than

368 droplets observed in control embryos using the same protocol (Fig. 4C and D). These large lipid-

369 containing structures were observable in live mutant embryos (Fig. 4J) but not in wild type

370 embryos (Fig. 4K) using Nomarski optics. Viewing through multiple focal planes in live,

371 developing embryos lacking W01A8.1 showed that these large lipid droplets are present in

372 embryos during the early mitotic divisions and were localized around the nucleus. Staining with

373 LipidTox (after fixation) confirmed the lipid content in the vesicular structures arranged around

374 dividing nucleus (Fig. 4L and M). These large vesicles persist through the two-cell stage,

375 disappearing in most embryos with more than 6 cells. On fixed embryos stained with LipidTox,

376 larger than wild type lipid droplets are visible until late embryonic stages, including three fold 377 embryos.

378 In order to visualize lipid-containing structures in W01A8.1 null mutants and in controls

379 in vivo, we used CARS microscopy (done with kind help from Dr. Zhongxiang Jiang, Leica

380 Microsystems, Mannheim, Germany). The CARS systems allow visualization of lipids of

381 specific categories by tuning into symmetric $\mathrm{CH}_{2}$ vibrations of specific fat composing molecules

382 (Zumbusch, Langbein \& Borri 2013). CARS microscopy clearly confirmed the formation of

383 large lipid containing vesicles in early embryos and allowed detailed analysis of the W01A8.1

384 null phenotype. CARS microscopy also confirmed the gradual increase of the size of lipid

385 containing structures during oogenesis (Fig. 5A and B), the sudden re-localization of these

386 structures to the periphery of the dividing nucleus in the first embryonic division (Fig. 5E), and 
387 the propagation of this phenotype, although with gradually diminishing appearance, throughout

388 embryonic development (Fig. 5F and H). CARS microscopy detected this phenomenon also in

389 wild type animals, although the size of lipid containing structures was smaller making the

390 phenomenon of the sudden re-localization of lipid containing structures less obvious (Fig. 5C, D

391 and G) than in W01A8.1 null embryos. In contrast to embryos, lipid-containing structures in

392 intestinal and epidermal cells of adult W01A8.1 null mutants (Fig. 5J and L) were smaller than

393 lipid-containing structures in control animals (Fig. 5I and K).

394 Morphometric analysis confirmed that W01A8.1 null mutant embryos contained larger

395 lipid positive structures recognized by CARS microscopy (Fig. 6A and B) that represent a larger

396 total area (Fig. 6C), as determined by quantitating individual focal planes images. Morphometry

397 revealed many small structures with area 1 to 4 AU (arbitrary units) with the provided threshold

398 settings (Fig. 6A and E). There was a clear inverse relation in the number of large and small

399 structures (with area $<5 \mathrm{AU}$ ) for embryos as well as for adult tissues. The analysis in Fig. 6B and

$400 \mathrm{~F}$ shows that inclusion of small structures into analysis does not significantly affect the results

401 but affects only standard deviation of the particle size distribution indicating that the results are

402 independent on the setting of the limit for the size of lipid containing structures. The probability

403 of the results were assayed using Student's t-test and found to be statistically significant as the

404 probability of this result, assuming the null hypothesis (no difference between control and 405 experimental sets) was less than 0.0001 .

406 Despite the fact that there were a larger number of individually recognizable lipid 407 containing structures in wild type embryos (Fig. 6D) the mean area of these structures in mutants 408 was considerably larger (Fig. 6C). In contrast, adult mutant animals contained smaller, more 
409 numerous lipid-containing structures (Fig. 6E, F and H) that covered a smaller total area (Fig.

$4106 \mathrm{G})$ (and therefore volume) compared to wild type controls.

411 The morphometric analysis confirms that there is more CARS positive signal and 412 therefore most likely more fat in W01A8.1 null embryos (despite lower threshold used for 413 analysis of wild type embryos) and less CARS positive signal (and less fat) in adult tissues of 414 W01A8.1 null animals compared to controls.

415 The analysis of the number of progeny laid by animals lacking W01A8.1 in comparison 416 to wild type animals showed a decrease of progeny in mutant animals statistically significant in 417 the day 3 (Fig. S5).

418

\section{Discussion}

Lipolysis is a tightly regulated cellular process in which triacylglycerol fatty acids

421 (TAG) are degraded into free fatty acids (FFA) and glycerol (G) with intermediates of 422 diacylglycerol (DAG) and monoacylglycerol (MAG). The function and regulation of three key 423 lipases (adipose triglyceride lipase (ATGL), hormone-sensitive lipase (HSL) and monoglyceride 424 lipase (MGL) have been studied in great detail in mammalian adipocytes (reviewed in (Lass et 425 al. 2011)). Multidomain and multifunctional LD coating proteins, the perilipins, mediate the access of ATGL and HSL to LDs. Briefly (Fig. 7), the phosphorylated N-terminal domain PAT 427 in perilipin interacts with HSL and brings it in contact with lipid droplets (LDs) (Shen et al. 428 2009). At the same time, the C-terminal phosphorylation (controlled by the kinase PKA) releases a specific activator of ATGL named ABHD5 without which ATGL remains inactive in the cytoplasm. The final step of the glycolysis is catalyzed by MGL. Conversely, unphosphorylated

431 perilipin blocks lipolysis in the basal fed state by blocking the access of lipolytic enzymes to the 
432 fat stored in LDs. Both HSL and ATGL are the rate-limiting enzymes needed for fatty acids

433 mobilization (Schweiger et al. 2006). A variation of this regulatory process, although less well

434 understood in detail, exists in other cells and organisms. Most organisms so far studied contain

435 several perilipin genes, complicating the analysis of complete perilipin loss-of-function.

436 Clear orthologues of ATGL, HSL, MGL, ABHD5 and catalytic and regulatory subunits

437 of PKA have been identified in C. elegans (ATGL-1, HOSL-1, LID-1, KIN-1, KIN-2

438 respectively (Lee et al. 2014; Xie \& Roy 2015) (Fig. 7). The MGL orthologue remains to be

439 identified but several un-annotated homologous proteins exist (Birsoy, Festuccia \& Laplante

440 2013). A recent careful and elegant study of ATGL function and regulation (Lee et al. 2014)

441 revealed that the process in $C$. elegans was almost identical to that found in mammalian

442 adipocytes. Even the degradation of ABHD5 in the proteasome (Dai et al. 2013) is mirrored in $C$.

443 elegans (Lee et al. 2014). The glaring difference in fat storage and metabolism seemed to be the

444 absence of perilipin in nematode genomes.

445 Here we have established that $C$. elegans possesses a close homologue of perilipin that

446 is intimately involved in the regulation of lipid metabolism. Although the sequence alignment of

447 C. elegans and human homologues of perilipin does not appear visually very informative, the

448 underlying evolutionary conserved homology is statistically very significant. Perilipin is a

449 scaffolding protein allowing co-evolution of interacting domains and divergence of non-docking

450 sequences. Thus the function can be conserved even with limited amino acid conservation

451 across species. This evolutionary plasticity was already apparent in the alignment of the human

452 perilipin paralogues where only the knowledge of the three-dimensional structure enabled

453 observations of the similarities in the C-terminal domains (Hickenbottom et al. 2004). The

454 nematode sequences have diverged beyond the point where pairwise comparisons used in routine 
455 searches can reveal homology, hence the difficulty in identifying the nematode orthologues.

456 Only rigorous statistical analysis of the hidden Markov profiles of a great number of diverse 457 sequences made it possible to identify the conserved domain composition.

458 The nematode perilipin-related protein W01A8.1 contains all three major perilipin 459 features: N-terminal PAT domain, amphipathic region composed of imperfect helical repeats and 460 C-terminal apolipoprotein-like four-helix bundle. In mammals, the first two domains are known 461 to be responsible for the interaction with HSL and LDs respectively and the ATGL interaction 462 region resides in the C-terminus following the bundle. The function of the bundle is still unclear 463 but its stability probably fine-tunes the solubility and the affinity to LDs (Brasaemle 2007). All 464 these functions will have to be investigated in the isoforms of W01A8.1 in the future. The 465 repeats are confirmed by analysis of internal homology using the HHrepID algorithm and the 466 helical composition by secondary structure prediction. The bundle appears not to be stabilized by $467 \beta$-sheets as in human Perilipin 3 as revealed by the absence of the homology in the C-terminal 468 region; the $\beta$-sheets are similarly absent in Perilipin 1.

469 Our findings are consistent with a proteomic study that found that W01A8.1b is among 470 the most abundant proteins associated with LDs (Zhang et al. 2012). Similarly, perilipins are 471 abundant proteins on mammalian LDs, although the distribution and proportion of the individual 472 isoforms changes depending on the cell type and metabolic state (Brasaemle et al. 2004). 473 Perilipins are widely used as general markers of LDs and it seems that W01A8.1a or b can be 474 exploited for the same purposes; human PLIN1 was recently proposed as a marker for LDs in $C$. 475 elegans (Liu et al. 2014).

476 Surprisingly, in laboratory conditions C. elegans can overcome the complete loss of the 477 perilipin-related protein W01A8.1, presumably by activating perilipin-independent lipid 
478 degradation. Previous work has shown that an additional lipid degradation pathway, autophagy,

479 was important for lipid metabolism in C. elegans (Lapierre et al. 2013), mammals (Singh et al.

480 2009) as well as in yeast (van Zutphen et al. 2014). Similarly in Drosophila, which has two

481 perilipins (plin1 and plin2), the double mutants are viable but have small lipid droplets. This

482 suggests that perilipins are required for growth or maintenance of lipid droplets, but are

483 dispensable for lipolysis (Beller et al. 2010; Bi et al. 2012). The abnormal LD behavior, but

484 viability, of W01A8.1 null animals strongly suggests a regulatory role for the nematode perilipin-

485 related protein in the regulation of fat metabolism that is similar to perilipins in other phyla.

486 Taking in account the opposing phenotypes of Drosophila plin1 and plin2 loss of function

487 regarding to lipid droplet size, it is intriguing to speculate that individual W01A8.1 splice forms

488 may support distinct functions as well. It is also possible that some functions of W01A8.1

489 protein forms may be related to a proposed ancestral protein acting differently than mammalian

490 perilipins as was suggested by Beller et al. (Beller et al. 2010). A possibility of the existence of

491 an ancestor protein with wider, less specific functionality may be also considered if such a

492 parallel to enzymatic activities of ancestral proteins (Hujova et al. 2005) is taken in account.

493 CARS microscopy allowed a detailed analysis of lipid containing structures in wild

494 type and in mutant animals in vivo. Detection of lipids in vivo showed that the lack of the

495 perilipin homologue affects the intracellular distribution of lipid droplets, which is in agreement

496 with the role of perilipin homologue LSD2 (PLIN2) in movement of lipid droplets in Drosophila

497 (Cohen 2005; Welte et al. 2005). Analysis of lipid-containing structures in developing embryos

498 and in adult tissues suggested that W01A8.1 protein forms are likely to act differently in

499 embryos than in adult tissues and lipid-containing structures in embryos are likely to differ from

500 those of adult tissues. The characteristic aggregation of lipid-containing structures around the 
501 embryonic nuclei clearly detected in C. elegans embryos by CARS microscopy are reminiscent

502 of lipid droplets recently reported to be a characteristic feature of cancer stem cells in colorectal

503 carcinomas (Tirinato et al. 2015).

504 Our results suggest that the previously accepted view of a perilipin-independent

505 nematode fatty acid flux of LDs needs to be revisited. Clearly we see evidence for perilipin-like

506 LD regulation that is evolutionarily conserved. Based on the results reported here, W01A8.1 is

507 renamed with permission from WormBase as plin-1 (Cel-plin-1 with species identifier). With

508 only a single gene and a toolbox of forward and reverse genetic approaches at hand, C. elegans

509 offers an opportunity to explore the exact role of perlipin-related factors in fat regulation

510 throughout development of many different somatic and germline cells. Exploitation of these

511 opportunities will likely reveal new levels of regulation and novel players in the complex and

512 vital regulation of fat in all organisms.

513

514 


\section{Acknowledgements}

517 The authors thank WormBase and NCBI for accessibility of data and bioinformatics support and

518 CGC for the N2 wild type strain. Authors thank Dr. Zhongxiang Jiang and Leica Microsystems

519 CMS GmbH (Mannheim, Germany) for CARS microscopy. Authors thank Dr. Sebastian

520 Honnen, and Reviewer 2 for valuable suggestions and corrections.

521 
523

524

525

526

527

528

\section{References}

Altschul SF, Madden TL, Schaffer AA, Zhang J, Zhang Z, Miller W, Lipman DJ. 1997. Gapped BLAST and PSI-BLAST: a new generation of protein database search programs. Nucleic Acids Res 25: 3389-402.

Beller M, Bulankina AV, Hsiao HH, Urlaub H, Jackle H, Kuhnlein RP. 2010. PERILIPINdependent control of lipid droplet structure and fat storage in Drosophila. Cell Metab 12: 521-32.

Bi J, Xiang Y, Chen H, Liu Z, Gronke S, Kuhnlein RP, Huang X. 2012. Opposite and

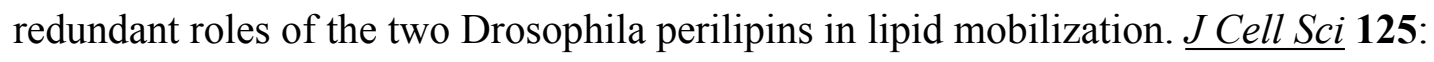
3568-77.

Biegert A, Soding J. 2008. De novo identification of highly diverged protein repeats by probabilistic consistency. Bioinformatics 24: 807-14.

Birney E, Clamp M, Durbin R. 2004. GeneWise and Genomewise. Genome Res 14: 988-95.

Birsoy K, Festuccia WT, Laplante M. 2013. A comparative perspective on lipid storage in

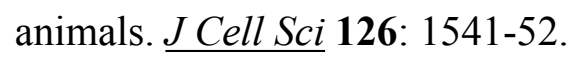

Brasaemle DL. 2007. Thematic review series: adipocyte biology. The perilipin family of structural lipid droplet proteins: stabilization of lipid droplets and control of lipolysis. $\underline{J}$ Lipid Res 48: 2547-59.

Brasaemle DL, Dolios G, Shapiro L and Wang R. 2004. Proteomic analysis of proteins associated with lipid droplets of basal and lipolytically stimulated 3T3-L1 adipocytes. $\underline{J}$ Biol Chem 279: 46835-42.

Brenner S. 1974. The genetics of Caenorhabditis elegans. Genetics 77: 71-94. 
546 Cohen RS. 2005. Microtubule motors: LSD2 trips the toggle. Curr Biol 15: R651-3.

547 Dai Z, Qi W, Li C, Lu J, Mao Y, Yao Y, Li L, Zhang T, Hong H, Li S, Zhou T, Yang Z,

$548 \quad$ Yang X, Gao G, Cai W. 2013. Dual regulation of adipose triglyceride lipase by pigment

549 epithelium-derived factor: a novel mechanistic insight into progressive obesity. $\underline{\mathrm{Mol} \text { Cell }}$

$550 \quad$ Endocrinol 377: 123-34.

551 Dickinson DJ, Ward JD, Reiner DJ, Goldstein B. 2013. Engineering the Caenorhabditis

552 elegans genome using Cas9-triggered homologous recombination. Nat Methods 10: 1028-

$553 \quad 34$.

554 Du X, Barisch C, Paschke P, Herrfurth C, Bertinetti O, Pawolleck N, Otto H, Ruhling H,

555 Feussner I, Herberg FW, Maniak M. 2013. Dictyostelium lipid droplets host novel

$556 \quad$ proteins. Eukaryot Cell 12: 1517-29.

557 Hickenbottom SJ, Kimmel AR, Londos C, Hurley JH. 2004. Structure of a lipid droplet

558 protein; the PAT family member TIP47. Structure 12: 1199-207.

559 Hujova J, Sikora J, Dobrovolny R, Poupetova H, Ledvinova J, Kostrouchova M, Hrebicek

560 M. 2005. Characterization of gana-1, a Caenorhabditis elegans gene encoding a single

561 ortholog of vertebrate alpha-galactosidase and alpha-N-acetylgalactosaminidase. $\underline{B M C}$

$562 \quad$ Cell Biol 6: 5.

563 Kimmel AR, Brasaemle DL, McAndrews-Hill M, Sztalryd C, Londos C. 2010. Adoption of

564 PERILIPIN as a unifying nomenclature for the mammalian PAT-family of intracellular

$565 \quad$ lipid storage droplet proteins. J Lipid Res 51: 468-71.

566 Kozusko K, Tsang VH, Bottomley W, Cho YH, Gandotra S, Mimmack M, Lim K, Isaac I,

567 Patel S, Saudek V, O'Rahilly S, Srinivasan S, Greenfield JR, Barroso I, Campbell

568 LV, Savage DB. 2015. Clinical and molecular characterization of a novel PLIN1 

frameshift mutation identified in patients with familial partial lipodystrophy. Diabetes $\mathbf{6 4 :}$ 299-310.

571

572

573

574

575

576

577

578

579

Lapierre LR, Silvestrini MJ, Nunez L, Ames K, Wong S, Le TT, Hansen M, Melendez A. 2013. Autophagy genes are required for normal lipid levels in C. elegans. Autophagy 9 : 278-86.

Lass A, Zimmermann R, Oberer M, Zechner R. 2011. Lipolysis - a highly regulated multienzyme complex mediates the catabolism of cellular fat stores. Prog Lipid Res 50: 14-27.

Lee JH, Kong J, Jang JY, Han JS, Ji Y, Lee J, Kim JB. 2014. Lipid droplet protein LID-1 mediates ATGL-1-dependent lipolysis during fasting in Caenorhabditis elegans. $\underline{\text { Mol Cell }}$ Biol 34: 4165-76.

Liu Z, Li X, Ge Q, Ding M, Huang X. 2014. A lipid droplet-associated GFP reporter-based screen identifies new fat storage regulators in C. elegans. J Genet Genomics 41: 305-13.

Lu X, Gruia-Gray J, Copeland NG, Gilbert DJ, Jenkins NA, Londos C, Kimmel AR. 2001. The murine perilipin gene: the lipid droplet-associated perilipins derive from tissuespecific, mRNA splice variants and define a gene family of ancient origin. $\underline{\text { Mamm }}$ Genome 12: 741-9.

Notredame C, Higgins DG, Heringa J. 2000. T-Coffee: A novel method for fast and accurate multiple sequence alignment. JMol Biol 302: 205-17.

O'Rourke EJ, Soukas AA, Carr CE, Ruvkun G. 2009. C. elegans major fats are stored in vesicles distinct from lysosome-related organelles. Cell Metab 10: 430-5.

Punta M, Coggill PC, Eberhardt RY, Mistry J, Tate J, Boursnell C, Pang N, Forslund K, Ceric G, Clements J, Heger A, Holm L, Sonnhammer EL, Eddy SR, Bateman A, Finn RD. 2012. The Pfam protein families database. Nucleic Acids Res 40: D290-301. 
592 Remmert M, Biegert A, Hauser A, Soding J. 2011. HHblits: lightning-fast iterative protein

593 sequence searching by HMM-HMM alignment. Nat Methods 9: 173-5.

594 Schweiger M, Schreiber R, Haemmerle G, Lass A, Fledelius C, Jacobsen P, Tornqvist H,

595 Zechner R, Zimmermann R. 2006. Adipose triglyceride lipase and hormone-sensitive

596 lipase are the major enzymes in adipose tissue triacylglycerol catabolism. J Biol Chem

$597 \quad 281: 40236-41$.

598 Shen WJ, Patel S, Miyoshi H, Greenberg AS, Kraemer FB. 2009. Functional interaction of 599 hormone-sensitive lipase and perilipin in lipolysis. JLipid Res 50: 2306-13.

600 Simeckova K, Brozova E, Vohanka J, Pohludka M, Kostrouch Z, Krause MW, Rall JE, 601 Kostrouchova M. 2007. Supplementary nuclear receptor NHR-60 is required for normal

Singh R, Kaushik S, Wang Y, Xiang Y, Novak I, Komatsu M, Tanaka K, Cuervo AM, embryonic and early larval development of Caenorhabditis elegans. Folia Biol (Praha) Czaja MJ. 2009. Autophagy regulates lipid metabolism. Nature 458: 1131-5.

Tabara H, Sarkissian M, Kelly WG, Fleenor J, Grishok A, Timmons L, Fire A, Mello CC. 1999. The rde-1 gene, RNA interference, and transposon silencing in C. elegans. Cell 99: 123-32.

Teixeira L, Rabouille C, Rorth P, Ephrussi A, Vanzo NF. 2003. Drosophila Perilipin/ADRP homologue Lsd2 regulates lipid metabolism. Mech Dev 120: 1071-81.

Timmons L, Court DL, Fire A. 2001. Ingestion of bacterially expressed dsRNAs can produce specific and potent genetic interference in Caenorhabditis elegans. Gene 263: 103-12.

613 Tirinato L, Liberale C, Di Franco S, Candeloro P, Benfante A, La Rocca R, Potze L, 
615

616

617

618

619

620

621

622

623

624

625

626

627

628

629

630

631

632

633

634

635

636

637

Carbone E, Todaro M, Medema JP, Stassi G, Di Fabrizio E. 2015. Lipid droplets: a new player in colorectal cancer stem cells unveiled by spectroscopic imaging. Stem Cells 33: 35-44.

van Zutphen T, Todde V, de Boer R, Kreim M, Hofbauer HF, Wolinski H, Veenhuis M, van der Klei IJ, Kohlwein SD. 2014. Lipid droplet autophagy in the yeast Saccharomyces cerevisiae. Mol Biol Cell 25: 290-301.

Vohanka J, Simeckova K, Machalova E, Behensky F, Krause MW, Kostrouch Z, Kostrouchova M. 2010. Diversification of fasting regulated transcription in a cluster of duplicated nuclear hormone receptors in C. elegans. Gene Expr Patterns 10: 227-36.

Wang C, St Leger RJ. 2007. The Metarhizium anisopliae Perilipin Homolog MPL1 Regulates Lipid Metabolism, Appressorial Turgor Pressure, and Virulence. $\underline{\text { J Biol Chem 282: }}$ 21110-5.

Welte MA, Cermelli S, Griner J, Viera A, Guo Y, Kim DH, Gindhart JG, Gross SP. 2005. Regulation of lipid-droplet transport by the perilipin homolog LSD2. Curr Biol 15: 126675.

Xie M, Roy R. 2015. The Causative Gene in Chanarian Dorfman Syndrome Regulates Lipid Droplet Homeostasis in C. elegans. PLoS Genet 11: e1005284.

Zhang P, Na H, Liu Z, Zhang S, Xue P, Chen Y, Pu J, Peng G, Huang X, Yang F, Xie Z, Xu T, Xu P, Ou G, Zhang SO, Liu P. 2012. Proteomic study and marker protein identification of Caenorhabditis elegans lipid droplets. Mol Cell Proteomics 11: 317-28.

Zumbusch A, Langbein W, Borri P. 2013. Nonlinear vibrational microscopy applied to lipid biology. Prog Lipid Res 52: 615-32. 


\section{1}

Identification of $C$. elegans protein W01A8.1a as a close homologue of vertebrate perilipin.

C. elegans protein W01A8.1a is compared with nematode homologues of pairwise sequence identity lower then $70 \%$ and with Plin2 and 3 from two diverse vertebrates. The three perilipin specific domains (indicated in red) were identified through homology with human Plin3. The six 11-mer repeats in W01A8.1a (positions 126-136, 137-147, 148-158, 159-169, 170-180 and 181-191) were established with HHrepID algorithm (Biegert \& Soding 2008). The $\mathrm{N}$-terminal PAT domain is thought to interact with HSL. The central domain consisting of imperfect 11-mer repeats forming amphipathic helices is responsible for the main affinity to LDs and the C-terminal domain containing an apolipoprotein-like 4-helix bundle probably plays an additional role in the affinity to LDs and is known to interact with ABHD5 in mammalian Plin1 and 3 (Brasaemle 2007). Alignment was done using T-coffee alignment of all available nematode sequences aligned with vertebrate Plin2 and 3 sequences in three iterations using ProfileAlign routine in MyHits suite (myhits.isb-sib.ch). Selected sequences from top to bottom: (Species, database identifier): Caenorhabditis elegans, Q23095; Strongyloides ratti, CACX01001972.1; Loa loa, E1G5Y0 and ADBU02007219.1; Haemonchus contortus, CDJ80228.1; Bursaphelenchus xylophilus, CADV01008520.1; Heterorhabditis bacteriophora, ES742365.1 and ACKM01001830.1; Ascaris suum, U1NU60; Homo sapiens 2, PLIN2_HUMAN; Homo sapiens 3, PLIN3_HUMAN; Latimeria chalumnae 2, H3AYCO; Latimeria chalumnae 3, GAAA01019375.1. Nucleotide sequences were translated with Wise2 program (Birney, Clamp \& Durbin 2004). Amino acid types are colored according to the Clustal scheme (jalview.org/help/html/colourSchemes/clustal.html). 


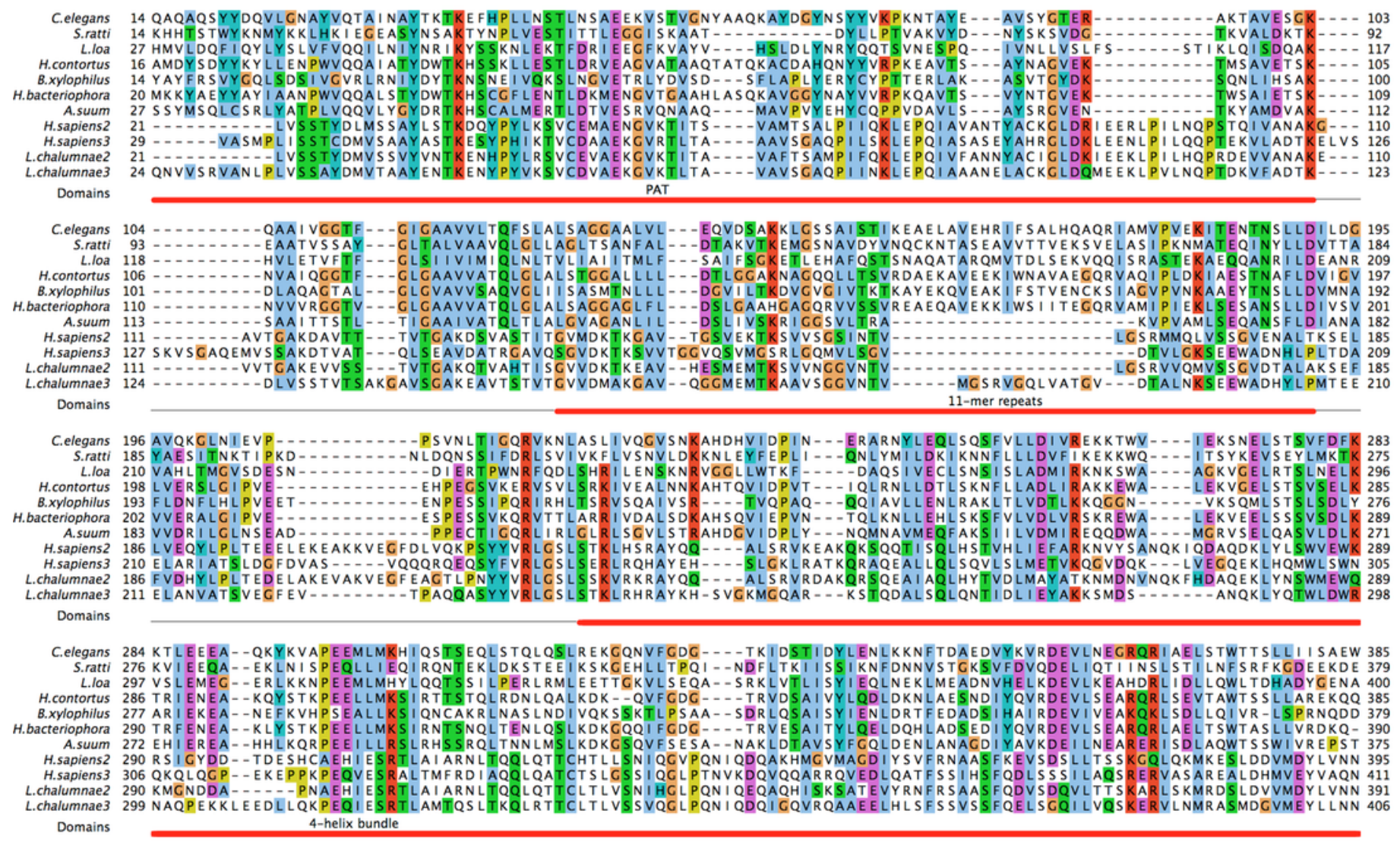




\section{2}

The expression W01A8.1::gfp reporter genes in transgenic strains.

W01A8.1a/c::GFP is shown in A, C, E, G, and I, and corresponding areas in Nomarski optics are shown in B, D, F, H and J. A) The onset of expression of W01A8.1a/c::GFP in epidermal cells and in intestinal cells of three-fold embryo. C) The expression of W01A8.1a/c::GFP in intestinal cells of an L2 larva. E and G) W01A8.1a/c::GFP expression in epidermal cells and intestinal cells of a young adult hermaphrodite. G) GFP fluorescence around lipid droplet-like structures in the intestine that are marked by arrows and arrowheads. Corresponding image in Nomarski optics is in H. I) A higher magnification the lipid droplet-like structures in epidermal cells labeled by W01A8.1a/c::GFP (shown in Nomarski optics in the J). K) Lipid droplets of an unfixed intestine labeled by W01A8.1b::GFP (corresponding Nomarski image is in $L$ ). $M, N$ and O) Part of the intestine of an adult larva expressing W01A8.1b::GFP (M) with corresponding staining of lipid droplets by LipidTox (N). O) LipidTox-positive lipid droplets (red) with W01A8.1b::GFP on the periphery (green) in this merged view. Bars represent 50 $\mu \mathrm{m}$ in $\mathrm{B}, \mathrm{H}, \mathrm{J}, \mathrm{L}$ and $\mathrm{N}$ and $100 \mu \mathrm{m}$ in $\mathrm{D}$ and $\mathrm{F}$. 

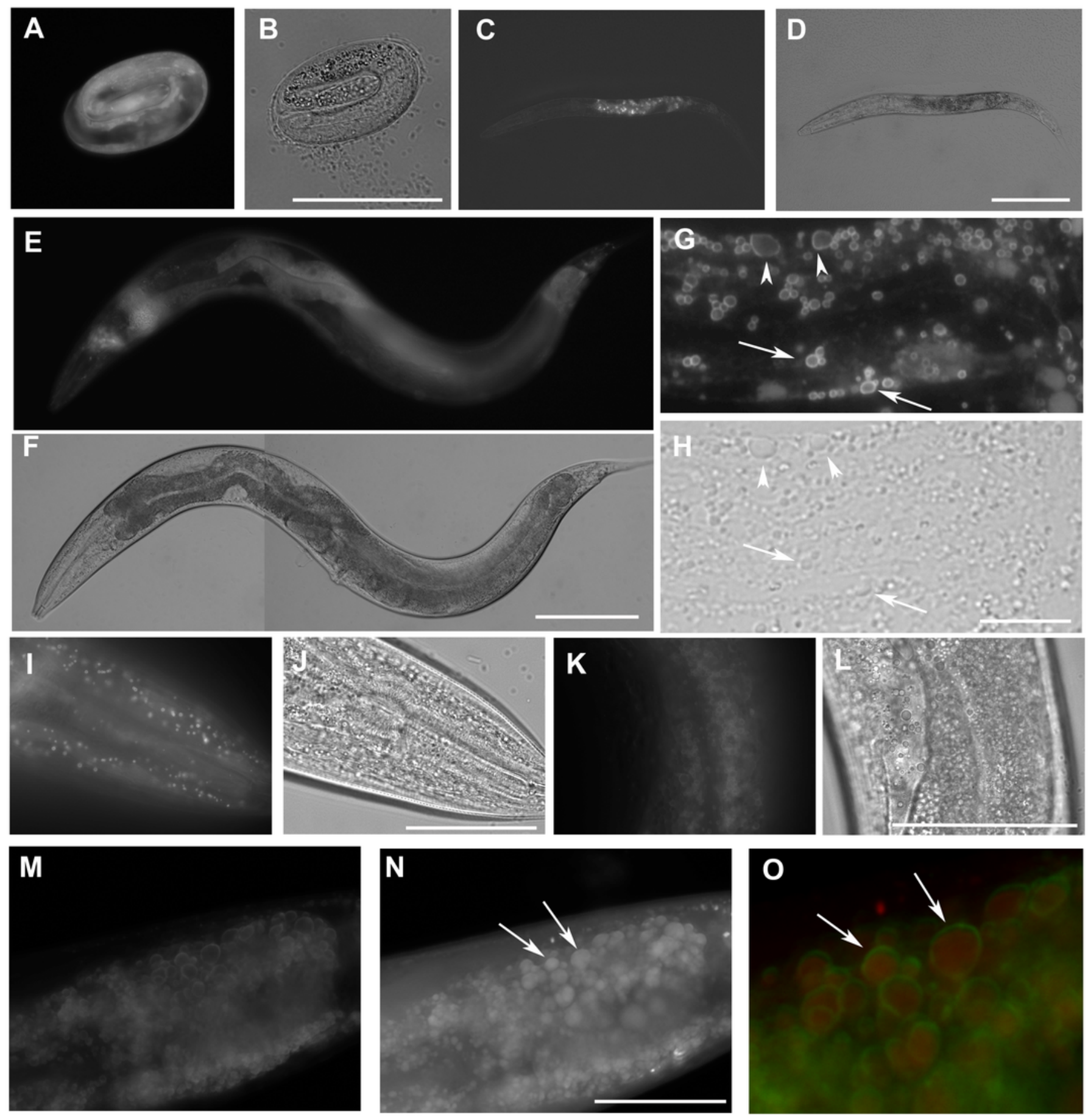


\section{3}

Expression of human perilipins fused to GFP in C. elegans.

A-C) Expression of human PLIN1::GFP in live transgenic C. elegans. PLIN1::GFP is localized on vesicles with an appearance of lipid droplets. PLIN2::GFP (D-F) is localized in transgenic animals on vesicular structures with an appearance of lipid droplets similarly as PLIN1::GFP. PLIN3::GFP (G, H and I) yields a more diffuse cytoplasmic pattern with faintly stained vesicular structures. A, D and $G$ and details in C, F and I show GFP in fluorescence microscopy and $B, E$ and $H$ corresponding areas to $A, D$ and $G$ in Nomarski optics. J, $K$ and $L$ show PLIN2::GFP in fluorescence microscopy (J) in fixed C. elegans stained with LipidTox (K). The area indicated by the white rectangle in $\mathrm{J}$ and $\mathrm{K}$ is magnified and merged for colocalization of PLIN2::GFP (green) and LipidTox (red) in L. Arrows indicate lipid droplets clearly marked by GFP with the LipidTox positive content. Bars represent $50 \mu \mathrm{m}$. 

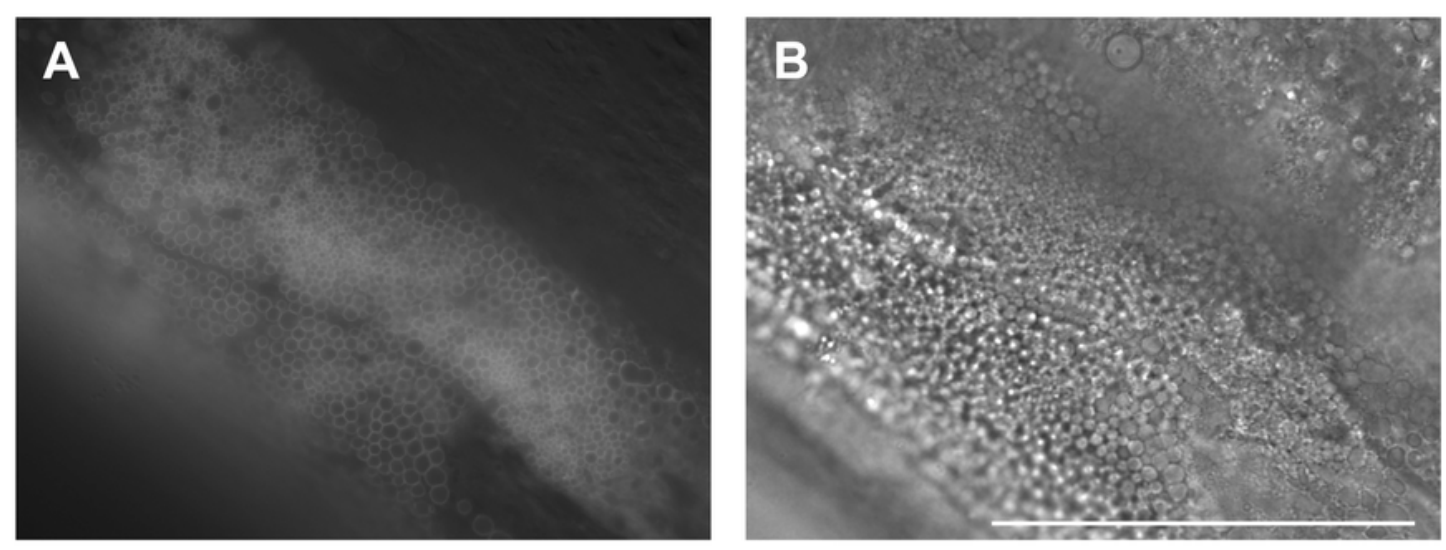

\section{PLIN1::GFP}
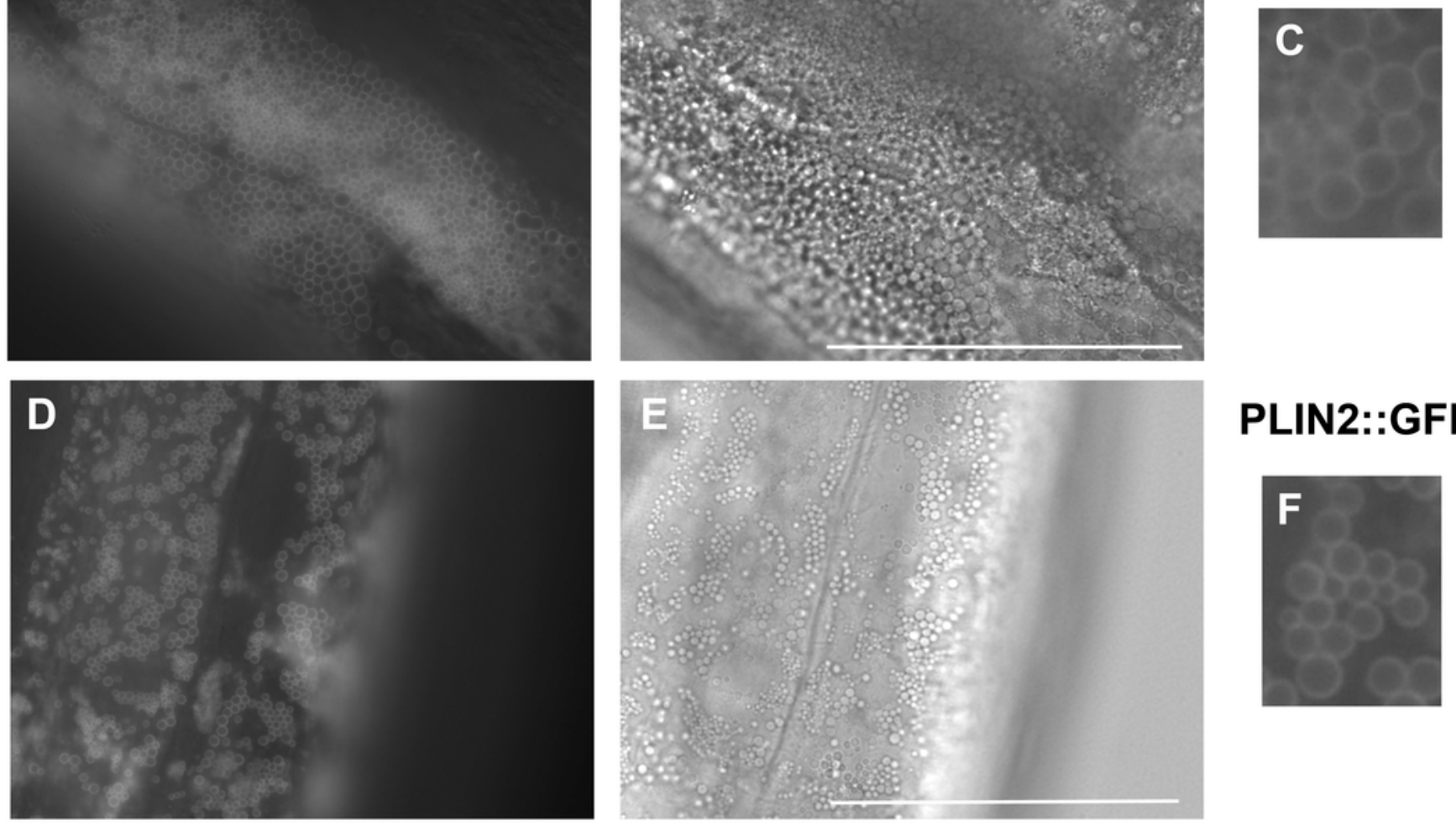

PLIN2::GFP

F

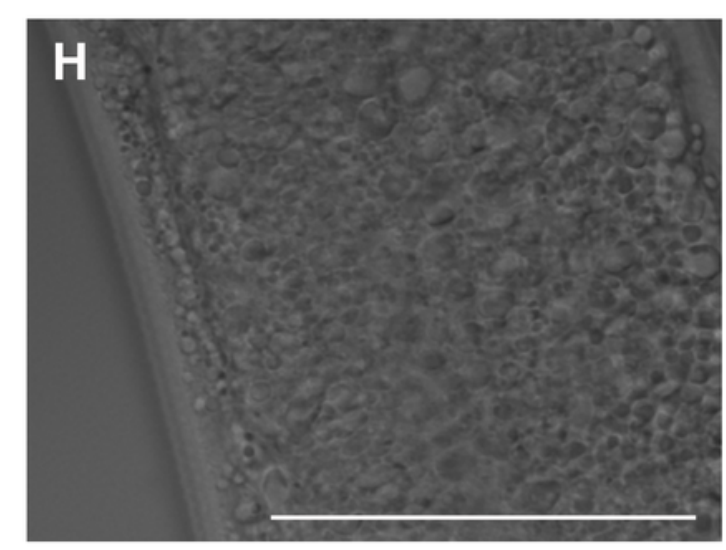

PLIN3::GFP
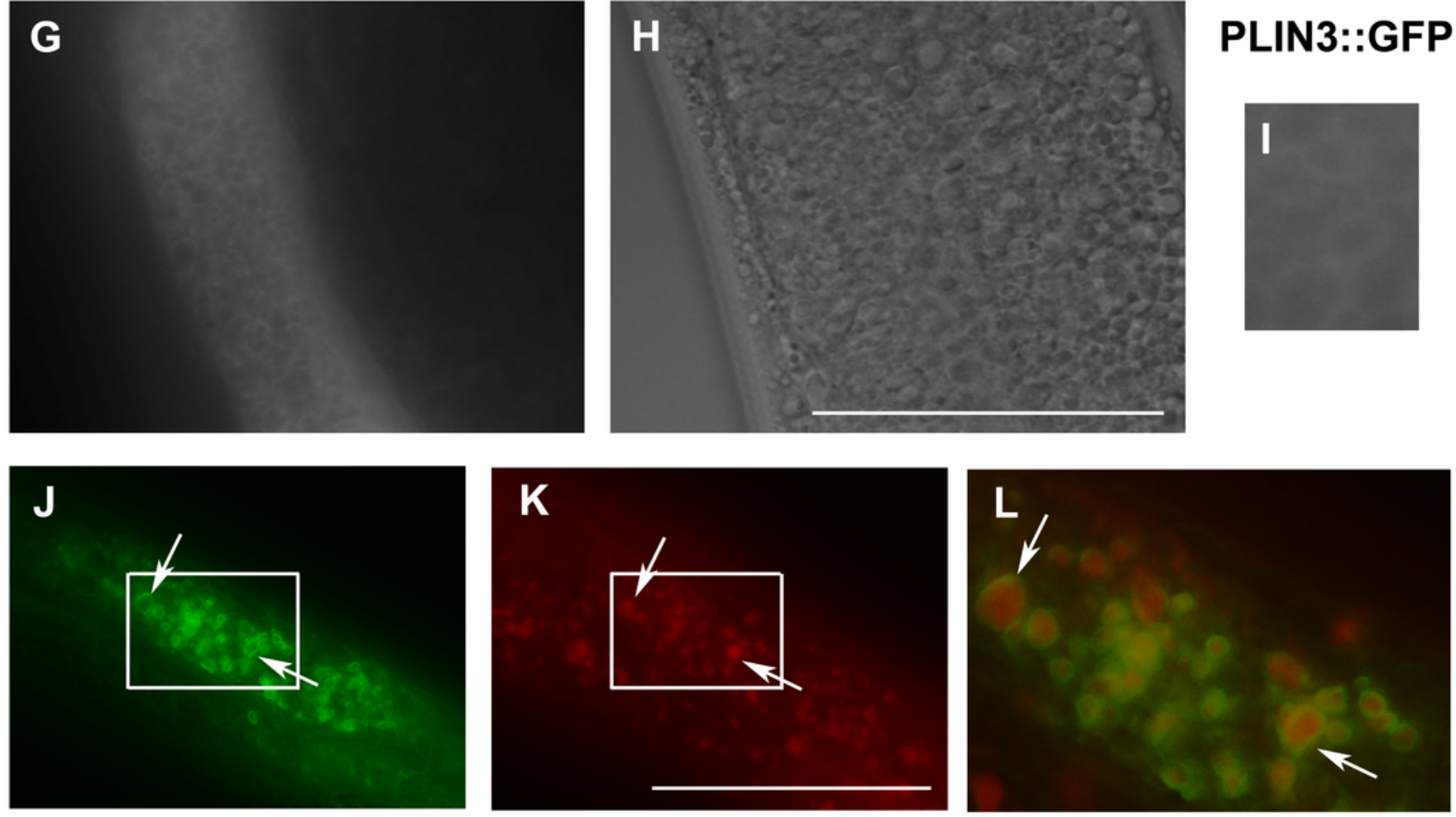


\section{4}

Loss of W01A8.1 function results in abnormal lipid droplet appearance.

A and B) An embryo from a hermaphrodite inhibited for W01A8.1 function by RNAi. Large lipid droplets stained by LipidTox (B) are visible also in Nomarski optics (A) in contrast with a control embryo that has only small and more evenly distributed lipid droplets (C - Nomarski optics and D - LipidTox staining). E-J, L and M) Images of structures observed in animals with disrupted W01A8.1. E and F show structures with the appearance of lipid droplets in the intestine of an animal with disrupted W01A8.1 balanced with the synthetic transgene W01A8.1(synth)::gfp. GFP tagged synthetic W01A8.1a is localized on lipid droplets-like vesicular structures $(E)$. $F$ shows the same area stained with LipidTox. $G$ shows in magnification a merged image of the area indicated by white rectangles in $E$ and $F$. Arrows indicate W01A8.1(synth)::GFP labeled lipid droplets (green) positive for lipids in LipidTox staining (red). $\mathrm{H}$ and I show an embryo of a parent with disrupted W01A8.1 that had confirmed loss of the balancing transgene. Large LipidTox stained droplets are visible in Nomarski optics (H) as well as in LipidTox staining (I). Their enlargement is clearly visible in comparison with the wild type embryo shown in panels $C$ and $D$. J and $K$ are images of live animals. J shows an embryo with disrupted W01A8.1 and confirmed loss of the balancing transgene. Large vesicular structures are formed around the dividing nucleus (arrows). $\mathrm{K}$ shows a control embryo with normal appearance of the nuclear periphery (arrow). L and $M$ show a one cell embryo from a parent with disrupted W01A8.1 and confirmed loss of extrachromosomal array containing W01A8.1(a)synth:: gfp after fixation and staining by LipidTox with large lipid droplets around the dividing nucleus visible in Nomarski optics (L) and positive for lipids in LipidTox staing (M) indicated by arrows). Bars represent $10 \mu \mathrm{m}$. 


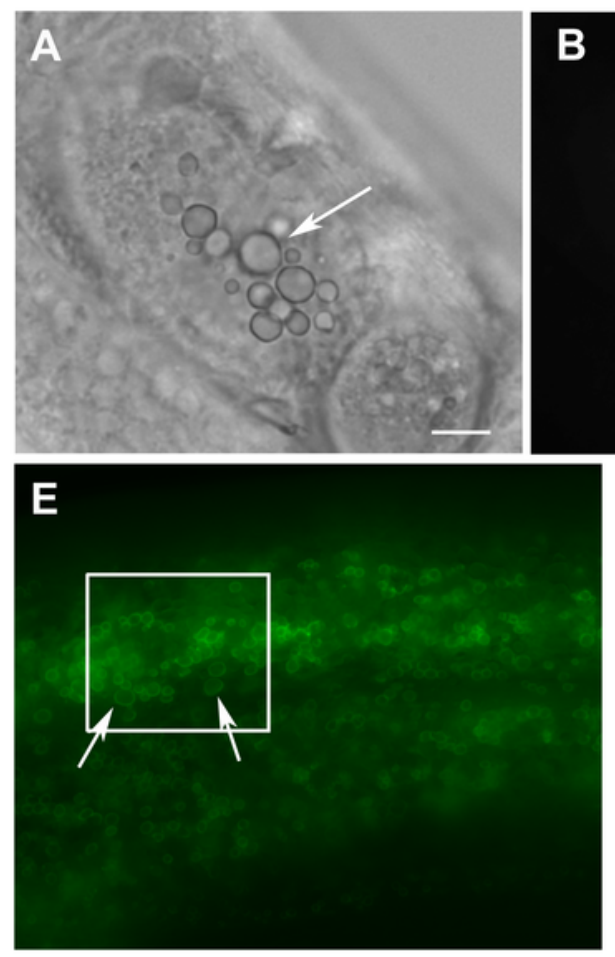

B
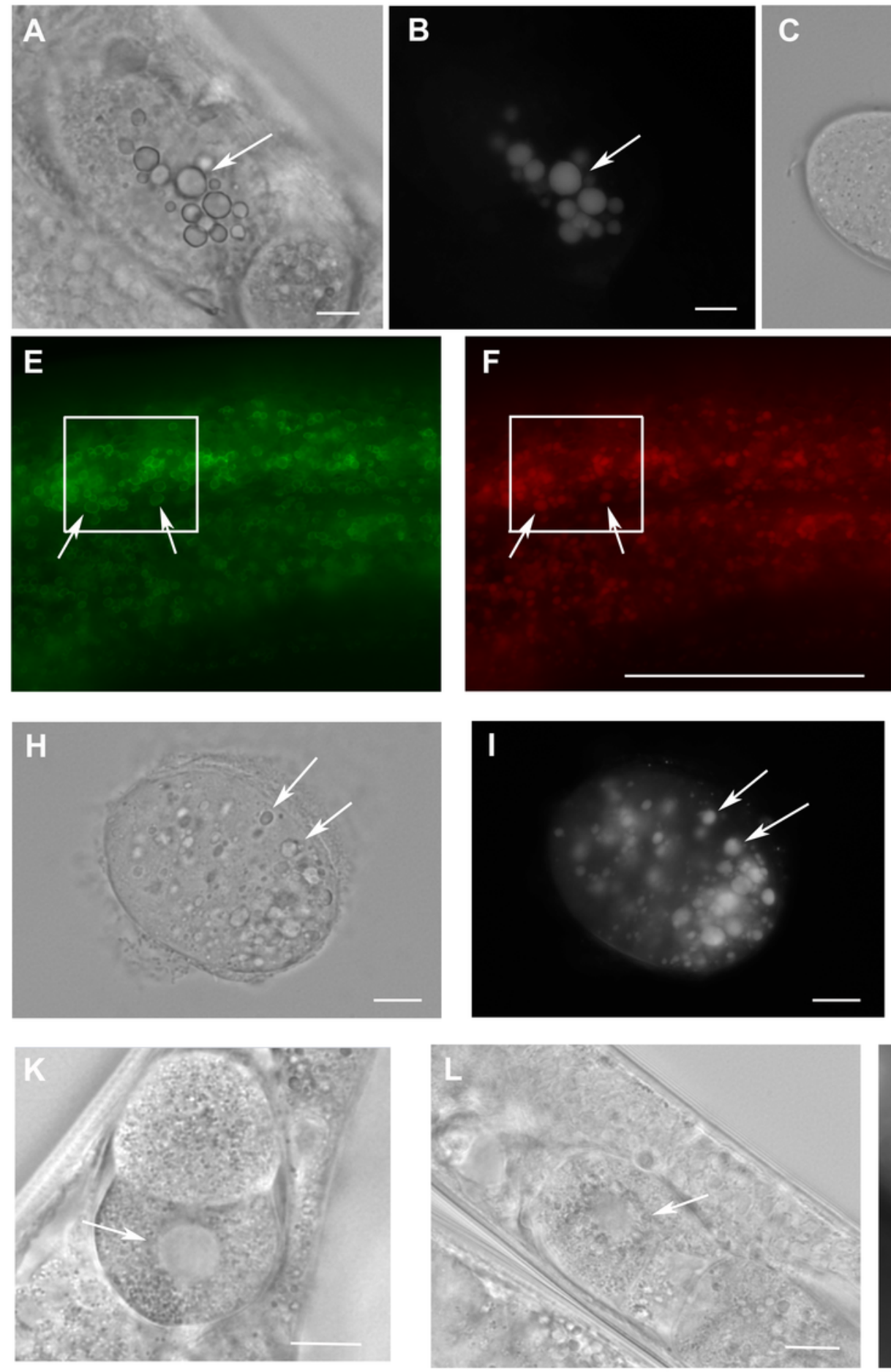

D

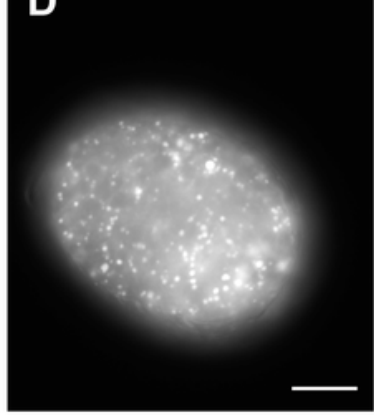

G
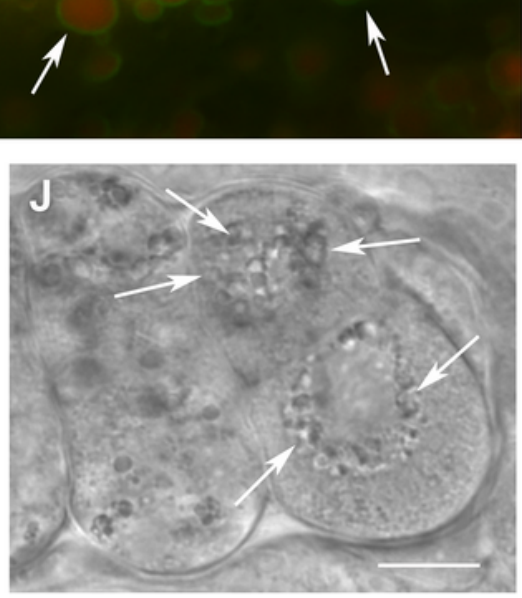

M

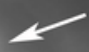




\section{5}

Analysis of lipid containing structures in live wild type animals and in animals with disrupted W01A8.1 by CARS microscopy.

CARS microscopy was performed using constant magnification and intensity settings (20\% laser intensity) with the exception of control embryos (panels $C$ and $G$ ) that were examined at $30 \%$ laser intensity since the lipid content was lower in wild type. The brightness of the entire figure was digitally enhanced using Adobe Photoshop brightness setting (+150 units) for better visibility of structures. Arrows indicate lipid containing structures in paired panels. A) The germline of a wild type adult hermaphrodite animal with small lipid containing structures in oocytes and an increase in their number and size during oogenesis. B) The germline of a mutant hermaphrodite animal. Lipid containing structures are bigger compared to the control animal yet distributed evenly in mutant oocytes. C) Lipid containing structures localize around the nucleus in one cell wild type embryos. This tendency of the association of the lipid containing structures with nuclear periphery can be seen also during later developmental stages in wild type embryos (D). E, F and H) Enlarged lipid containing structures arranged around the nuclei in mutant embryos. F) The formation of clusters of lipid containing structures on the periphery of nuclei. G) shows a wild type embryo at later stage of the development. H) The lipid containing structures progressively diminish in size in mutant embryos during later stages of embryonic development (arrowhead). I and J) Lipid containing structures in enterocytes of wild type (I) and mutant (J) animals. In contrast to embryos, which exhibit higher CARS signal and bigger lipid containing structures in mutant animals, gut cells in adult animals show the opposite, that is, a reduced fat-related CARS signal and smaller lipid containing structures in mutant animals. Similarly, lipid containing structures in epidermal cells (panels $\mathrm{K}$ and $\mathrm{L}$ ) are bigger in wild type animals as shown in the panel $\mathrm{K}$ and smaller in mutant animals (L). Bars represent $50 \mu \mathrm{m}$. 

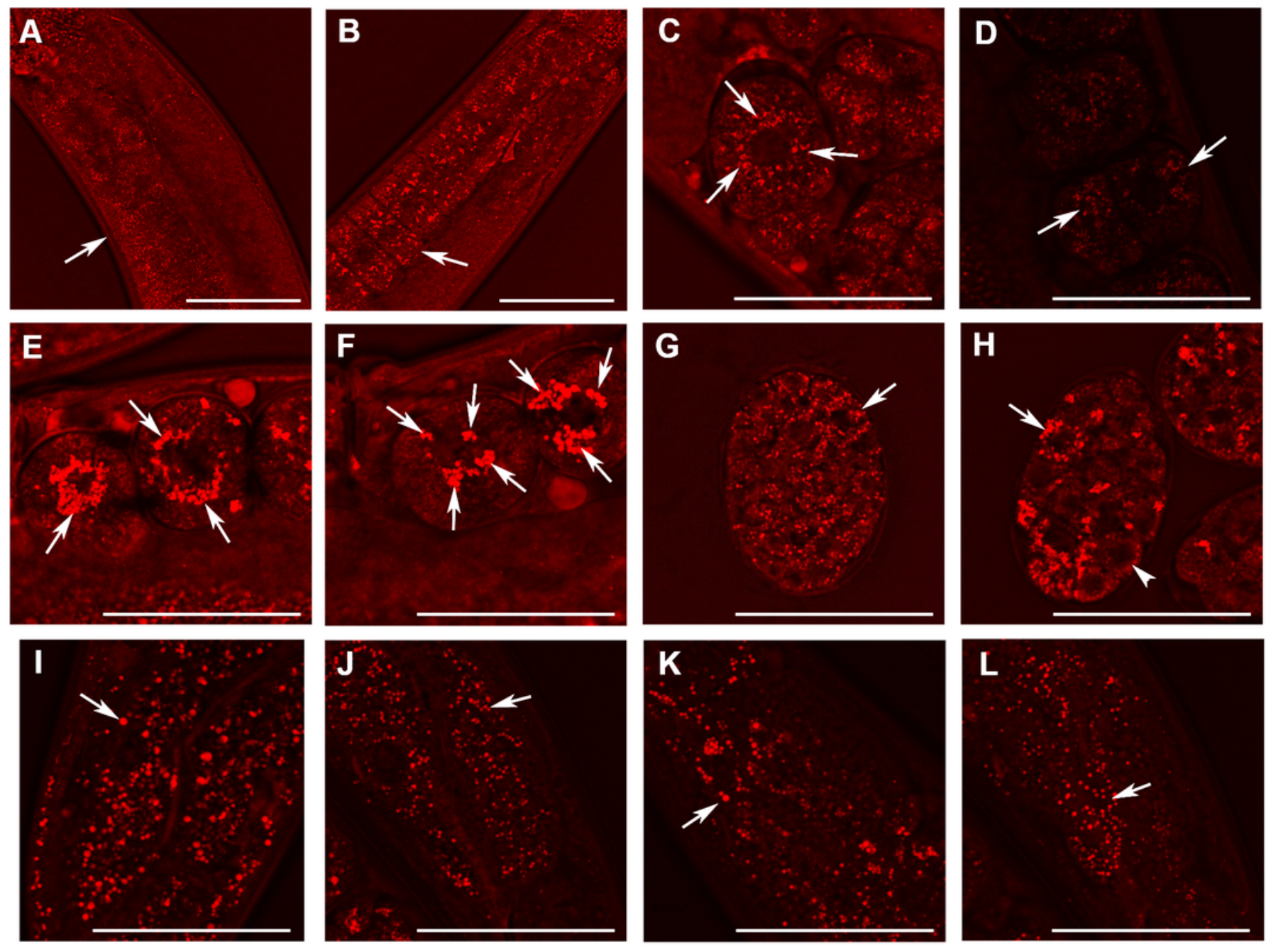
6

Morphometric analysis of lipid containing structures by CARS microscopy in wild type and W01A8.1 null animals.

Morphometric analysis was performed on CARS positive structures in single focal plane images acquired from representative mutant and wild type animal image stacks. Panels A to D compare early embryos from mutant (MUT) and wild type (WT) animals using seven representative CARS images. Panel A shows the mean area of all individually recognizable structures and panel B shows the mean area of all individually recognizable structures with an area bigger or equal to 5 arbitrary units (derived from pixels at the same settings). Panel C compares the total area of all CARS positive structures in mutant and wild type embryos while panel D compares the total number of individually recognizable CARS positive structures (lipid containing structures - LCS) in the same embryos. Panels E to H compare adult somatic tissue (tail region) from mutants and wild type hermaphrodites using five representative CARS images. Panel E shows the mean area of all individually recognizable CARS positive structures and panel $F$ shows the mean area of all individually recognizable structures with an area bigger or equal to 5 arbitrary units. Panel $\mathrm{G}$ compares the total area of all CARS positive structures in mutant and wild type tail regions while panel $\mathrm{H}$ compares the total number of individually recognizable CARS positive structures (LCS) in the same regions. Vertical bars in $A, B, E$ and $F$ represent Standard Deviation. The results presented in panels A, B, E and F are statistically significant in two-tailed Student's t-test $(p<0.0001)$. 

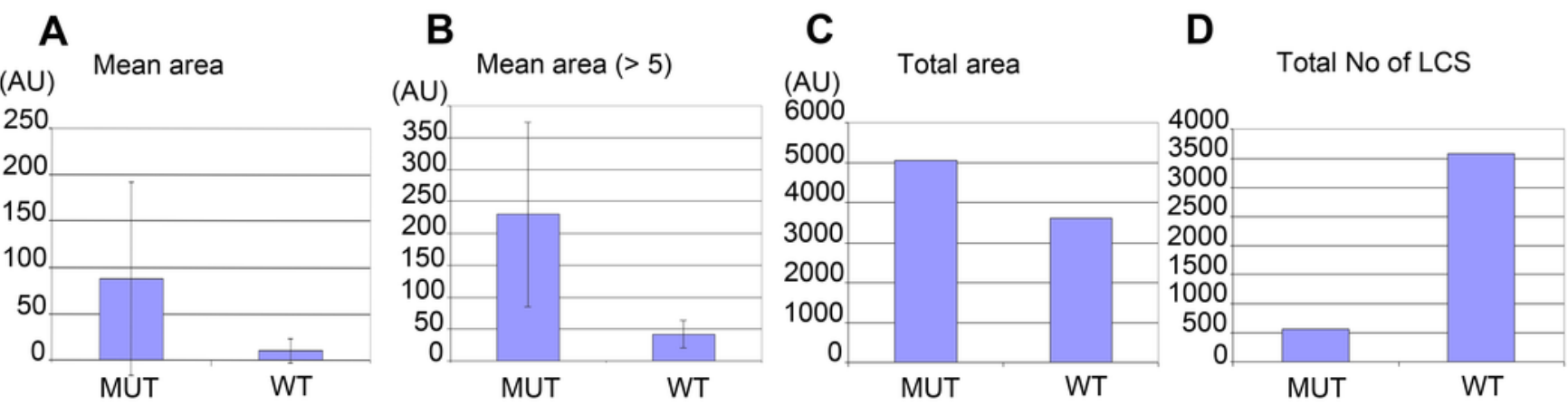

E

\section{F}

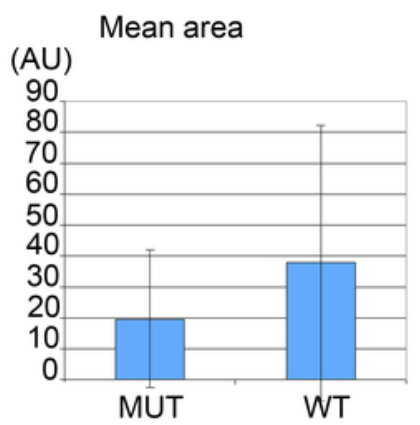

G

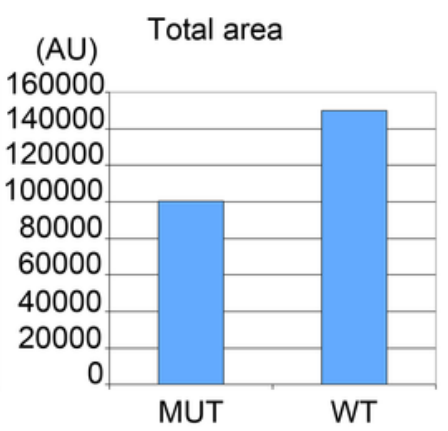

\section{H}

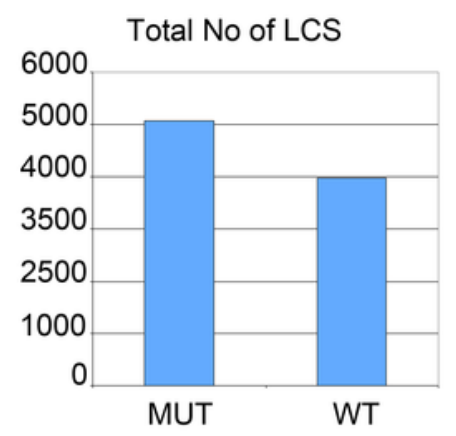




\section{7}

Enzymes and regulatory proteins involved in lipolysis (Adapted from (Lass et al. 2014).

Mammalian proteins are indicated above the arrows and their $C$. elegans orthologues (Lee et al. 2014) below. Triacylglycerol (TAG) is progressively hydrolysed to diacylglycerol (DAG), monoacylglycerol (MAG) and glycerol (G) by lipases specific for each of these steps: adipose triacylglycerol lipase (ATGL), hormone-sensitive lipase (HSL) and finally monoacylglycerol lipase (MGL). LID-1 and C37H5.3 were proposed to be orthologues of ABHD5/CGI58 in C. elegans (Lee et al. 2014, Xie \& Roy 2015). HSL also shows some activity in the first and third step. The access of ATGL and HSL to lipid droplets is regulated by perilipin, which is under the control of protein kinase A (PKA). W01A8.1 is proposed to be a perilipin orthologue in the present work.

\section{Mammals}

C. elegans

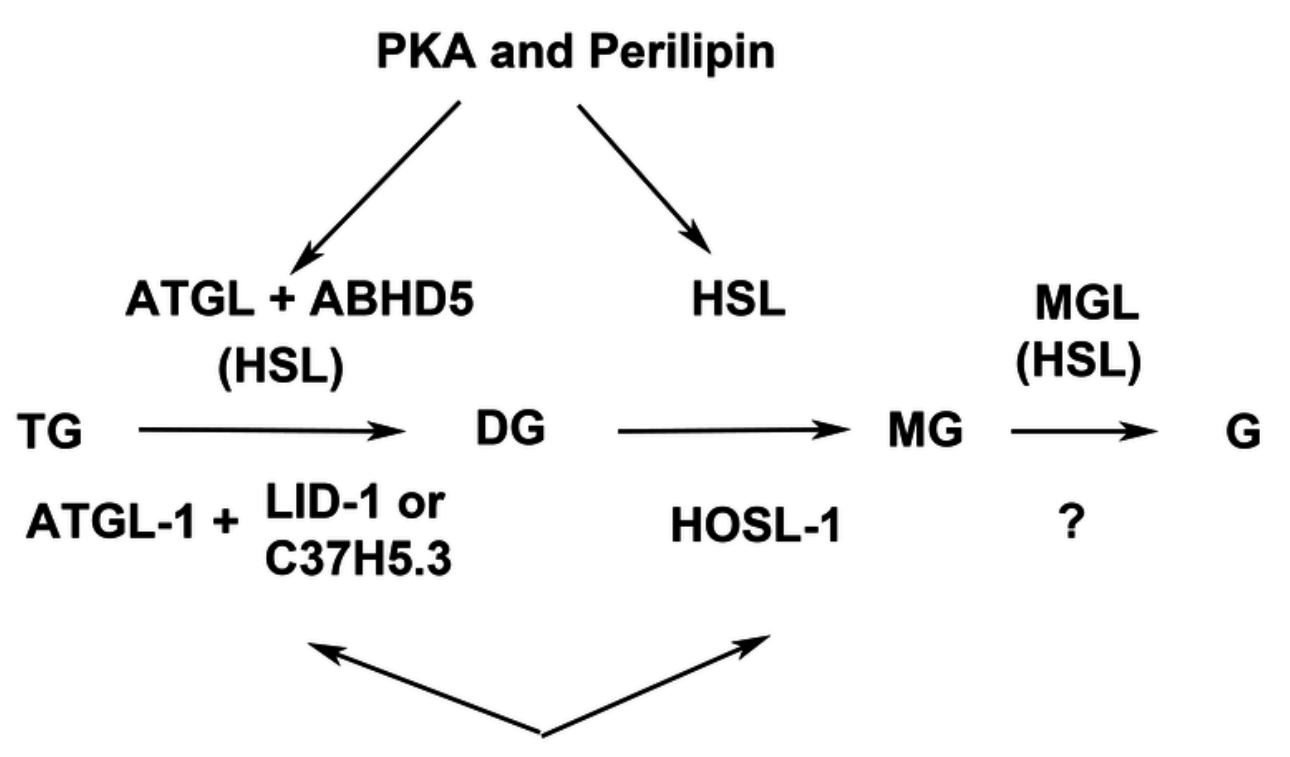

KIN-1/KIN-2 + PLIN-1 (W01A8.1) 